\title{
Tumorigenesis: A Mitochondrial Story
}

\section{Sharma LR, Milstein AC and Bai $\mathbf{Y}^{*}$}

Department of Cellular and Structural Biology, University of Texas Health Science Center at San Antonio, San Antonio, Texas 78229, USA

Cancer presents the scientific community with a complex problem, leading to difficult treatment approaches. This owes mainly to the high degree of genetic variability among cancer types and their metabolic adaptation for limiting nutrient and oxygen conditions. In recent years we have witnessed growing progress in understanding the critical role of mitochondrial alterations in tumorigenesis. Mitochondria play a central role in regulating cell death and proliferation. Mitochondrial DNA (mtDNA) mutations are common in cancer cells and pathophysiological consequences of mtDNA mutations are providing new insights into importance and complexity of mitochondrial mechanisms in tumorigenesis. Here we summarize the emerging views on mtDNA and mitochondrial regulation in cancer occurrence and development.

\section{The Brief History of Warburg Hypothesis}

Cancer cell growth is tightly correlated with altered cellular metabolism. The early observation that cancer cells relied on less energy production efficient glucose fermentation rather than respiratory metabolism even in the presence of oxygen was the basis for the Warburg Hypothesis in which the cancer was proposed as the consequence of injury to respiration [1]. Subsequently, abnormal mitochondrial morphology and activities had been reported in various cancer types. However, it was later widely accepted that mitochondrial defects are rare and that most tumors retain the capacity for oxidative phosphorylation and consume oxygen at rates similar to those observed in normal tissues [2]. In fact, mitochondrial functions such as ROS generation are crucial for transformation in some systems [3]. It has since been proposed that glycolytic metabolism arises as an adaptation to hypoxic conditions during the early avascular phase of tumor development, as it allows for ATP production in the absence of oxygen. Adaptation to the resulting acidic microenvironment that is caused by excess lactate production may further drive the evolution of the glycolytic phenotype. Most recently, it has been proposed that aerobic glycolysis provides a biosynthetic advantage for tumor cells, and allows effective shunting of carbon to key subsidiary biosynthetic pathways. This reprogramming of cellular metabolism toward macromolecular synthesis is critical to supplying enough nucleotides, proteins, and lipids for cell proliferation rather than providing ATP. Therefore, contrary to Warburg hypothesis, it is now becoming clearer that cancer cells do not inactivate mitochondrial energy metabolism, rather alter mitochondrial bioenergetics and biosynthesis state.

\section{MtDNA Haplogroups Modulating Cancer Occurrence}

A human mtDNA haplogroup is defined by unique sets of mtDNA polymorphisms, reflecting mutations accumulated by a discrete lineage during evolution. These haplogroups have been implicated in various human conditions including longevity, neurodegenerative diseases, infectious diseases and increased cancer risk [4]. However, so far most studies have been largely limited to population and epidemiology investigations; it is still unclear whether mtDNA haplogroups contribute to the occurrence of a specific cancer type. We carried out a comprehensive case-controlled study of mtDNA from large cohorts of patients with colorectal $(n=108)$ and breast $(n=104)$ cancer and found that patients with mtDNA haplogroup $M$ exhibited increased risk of breast cancer occurrence, and this risk was even more pronounced in a sub-haplogroup of $\mathrm{M}$ which is D5 [5]. This study indicates that mitochondrial haplogroups could have a cancer-specific role in modulating cancer development. This line of investigation shows promise and could lay the groundwork for personalized medicine and advanced diagnostics.

\section{Mitochondrial Dysfunction Regulating Tumorigenesis and Metastasis}

With the availability of advanced sequencing methods, mutations in both the non-coding and coding regions of the mtDNA have been identified in various types of human cancers. One of the first studies analyzing mtDNA in cancer cells demonstrated that among 10 colorectal cancer lines, seven of them exhibited mutations in their mtDNA content including complex I subunit genes [6]. Since mitochondrial complex I is the entry point for electrons in respiratory chain and major ROS generation site, more mutations were found in complex I subunit genes [7]. These ROS generating complex I mutation contributes to tumor progression by enhancing the metastatic potential of tumor cells [8]. Conversely, it has also been hypothesized that non ROS-producing mtDNA mutations can also promote tumor metastasis through altering membrane potential and activating PI3K/AKT pathway [9]. We examined the contribution of one of the colorectal cancer specific mtDNA mutation: complex I subunit 5 gene (ND5) on mitochondrial dysfunction and tumorigenesis. We found that with increasing mutant ND5 mtDNA content, mitochondrial functions declined progressively, whereas lactate production and dependence on glucose increased. Interestingly, cell line carrying the heteroplasmic mutation in complex I showed enhanced tumor growth [10]. These complex I dysfunction alters the redox ratio (NADH/NAD) and activates AKT signaling pathway which enhances tumorigenesis [11]. Altogether these studies implicate a critical role of complex I defects in tumorigenesis.

\section{Mitochondrial TCA Cycle Components in Tumorigenesis}

The role of mitochondrial metabolism came to attention since mutations in nuclear genes of several key enzymes of TCA cycle including Isocitrate dehydrogenase (IDH), Succinate dehydrogenase $(\mathrm{SDH})$ and Fumarate hydratase $(\mathrm{FH})$ have been identified in various tumors [12-14]. In addition to TCA cycle, mitochondria are involved in

*Corresponding author: Bai Y, Department of Cellular and Structural Biology, University of Texas Health Science Center at San Antonio, 7703 Floyd Cur Drive, San Antonio, Texas 78229, USA, Tel: 1-210-567-0561; Fax: 1-210-5673803; E-mail: baiy@uthscsa.edu

Received November 21, 2012; Accepted November 22, 2012; Published November 28, 2012

Citation: Sharma LR, Milstein AC, Bai Y (2012) Tumorigenesis: A Mitochondrial Story. J Carcinogene Mutagene 3: e109. doi:10.4172/2157-2518.1000e109

Copyright: (c) 2012 Sharma LR, et al. This is an open-access article distributed under the terms of the Creative Commons Attribution License, which permits unrestricted use, distribution, and reproduction in any medium, provided the original author and source are credited. 
Citation: Sharma LR, Milstein AC, Bai Y (2012) Tumorigenesis: A Mitochondrial Story. J Carcinogene Mutagene 3: e109. doi:10.4172/21572518.1000e109

Page 2 of 2

several other key metabolic processes such as fatty acid oxidation and amino acid metabolism, which have also been altered in cancer cells. For example, glycine consumption and expression of the mitochondrial glycine biosynthetic pathway were strongly correlated with rates of proliferation across different cancer cell types [15]. Similarly, Fatty acid (FA) synthesis occurs at very high rates in tumor cells, which supports cancer growth by increasing availability of building blocks for cell membranes and for lipids containing molecules involved in cell signaling. It has shown recently that even with defective mitochondria, tumor cell generates TCA cycle intermediates through glutamine dependent reductive carboxylation, which generate macromolecule precursors and support cancer cell growth [16].

\section{The New Mitochondrial Roles of Old Molecules Controlling Tumorigenesis}

Although, several onco-proteins such as PI3K/AKT, MYC, and tumor suppressor $\mathrm{p} 53$ are known to alter oncogenic signaling in cancer cells, their role in mitochondrial energy metabolism is now emerging. For example, PI3K/AKT activation enhances glycolysis by increasing glucose transporters, lipolysis and stimulating glycolytic enzymes; it also facilitates the diversion of glycolysis regulator: mitochondrial citrate from TCA cycle to acetyl CoA by activating ACL enzyme [17]. Conversely, cells harboring mtDNA mutations accumulate redox molecules such as NADH which causes PTEN inhibition and Akt activation in cancer cells [18]. Increased levels of MYC are seen in many aggressive tumors and it regulates mitochondrial oxygen consumption and mitochondrial biogenesis by enhancing transcriptional induction of TFAM and complex I genes [19]. The tumor suppressor $\mathrm{p} 53$ favors ATP production by OXPHOS and decreases the cellular ROS production by inducing TP53-Induced Glycolysis and Apoptosis Regulator (TIGAR) [20]. p53 also up-regulates OXPHOS complex IV by the induction of the Cytochrome c Oxidase (COX) Cu2+ chaperone, SCO2 [21]. Rapid cell proliferation in cancer results in a surge of oxygen consumption and thus, tumor tissues suffer from hypoxia. The transcription factor, HIF-1 (Hypoxia inducible factor-1), is the key mediator of the hypoxia response and affects mitochondria by inhibiting Pyruvate dehydrogenase $(\mathrm{PDH})$ and diverts the carbon flux from pyruvate into lactate and impairs mitochondrial machinery [22]. Overall, activation of oncogenes and/or inactivation of tumor suppressor genes favor glycolysis and alters the mitochondrial metabolism by rerouting the TCA intermediates for macromolecular synthesis required for growth and proliferation.

\section{Future Perspective}

Analysis of the physiological consequences of these mutations/ alterations is providing insight into how changes in mitochondrial metabolism can reprogram the nucleus and cell functions. However, many mysteries remain unsolved in our understanding of the role of mitochondria in normal vs cancer cells. In particular: Is the information on mitochondrial haplogroups in the mitochondrial genome useful for identifying individuals who are at risk for certain cancer types? Can mutations in mtDNA (complex I genes, for example) prognosticate specific types of tumorigenesis or malignant transformation? Are there unique mtDNA variations/mutations associated with specific types of cancer? Are there specific metabolic changes associated with specific mtDNA mutations in particular cancer type? Amid all these questions, one thing has become clear: mitochondria play a central role in cancer development. Further investigation in this area would not only provide a mechanistic link from mitochondria to tumorigenesis, but also help to develop a new metabolic biomarker and targeted therapies for cancer treatment.

\section{References}

1. Warburg O (1956) On respiratory impairment in cancer cells. Science 124: 269270

2. Weinhouse S (1976) The Warburg hypothesis fifty years later. Z Krebsforsch Klin Onkol Cancer Res Clin Oncol 87: 115-126.

3. Weinberg F, Hamanaka R, Wheaton WW, Weinberg S, Joseph J, et al. (2010) Mitochondrial metabolism and ROS generation are essential for Kras-mediated tumorigenicity. Proc Natl Acad Sci USA 107: 8788-8793.

4. Wallace DC (2005) A mitochondrial paradigm of metabolic and degenerative diseases, aging, and cancer: a dawn for evolutionary medicine. Annu Rev Genet 39: 359-407.

5. Fang H, Shen L, Chen T, He J, Ding Z, et al. (2010) Cancer type-specific modulation of mitochondrial haplogroups in breast, colorectal and thyroid cancer. BMC Cancer 10: 421

6. Polyak K, Li Y, Zhu H, Lengauer C, Willson JK, et al. (1998) Somatic mutations of the mitochondrial genome in human colorectal tumours. Nat Genet 20: 291293.

7. Lu J, Sharma LK, Bai Y (2009) Implications of mitochondrial DNA mutations and mitochondrial dysfunction in tumorigenesis. Cell Res 19: 802-815.

8. Ishikawa K, Takenaga K, Akimoto M, Koshikawa N, Yamaguchi A, et al (2008) ROS-generating mitochondrial DNA mutations can regulate tumor cell metastasis. Science 320: 661-664.

9. Kulawiec M, Owens KM, Singh KK (2009) Cancer cell mitochondria confer apoptosis resistance and promote metastasis. Cancer Biol Ther 8: 1378-1385.

10. Park JS, Sharma LK, Li H, Xiang R, Holstein D, et al. (2009) A heteroplasmic, not homoplasmic, mitochondrial DNA mutation promotes tumorigenesis via alteration in reactive oxygen species generation and apoptosis. Hum Mol Genet 18: 1578-1589.

11. Sharma LK, Fang H, Liu J, Vartak R, Deng J, et al. (2011) Mitochondria respiratory complex I dysfunction promotes tumorigenesis through ROS alteration and AKT activation. Hum Mol Genet 20: 4605-4616.

12. Baysal BE, Ferrell RE, Willett-Brozick JE, Lawrence EC, Myssiorek D, et al. (2000) Mutations in SDHD, a mitochondrial complex II gene, in hereditary paraganglioma. Science 287: 848-851.

13. Tomlinson IP, Alam NA, Rowan AJ, Barclay E, Jaeger EE, et al. (2002) Germline mutations in $\mathrm{FH}$ predispose to dominantly inherited uterine fibroids, skin leiomyomata and papillary renal cell cancer. Nat Genet 30: 406-410.

14. Yan H, Parsons DW, Jin G, McLendon R, Rasheed BA, et al. (2009) IDH1 and IDH2 mutations in gliomas. N Engl J Med 360: 765-773.

15. Jain M, Nilsson R, Sharma S, Madhusudhan N, Kitami T, et al. (2012) Metabolite profiling identifies a key role for glycine in rapid cancer cell proliferation. Science 336: 1040-1044.

16. Mullen AR, Wheaton WW, Jin ES, Chen PH, Sullivan LB, et al. (2012) Reductive carboxylation supports growth in tumour cells with defective mitochondria Nature 481: 385-388

17. Bauer DE, Hatzivassiliou G, Zhao F, Andreadis C, Thompson CB (2005) ATP citrate lyase is an important component of cell growth and transformation. Oncogene 24: 6314-6322.

18. Pelicano H, Xu RH, Du M, Feng L, Sasaki R, et al. (2006) Mitochondrial respiration defects in cancer cells cause activation of Akt survival pathway through a redox-mediated mechanism. J Cell Biol 175: 913-923.

19. Li F, Wang Y, Zeller KI, Potter JJ, Wonsey DR, et al. (2005) Myc stimulates nuclearly encoded mitochondrial genes and mitochondrial biogenesis. Mol Cell Biol 25: 6225-6234.

20. Bensaad K, Tsuruta A, Selak MA, Vidal MN, Nakano K, et al. (2006) TIGAR, a p53-inducible regulator of glycolysis and apoptosis. Cell 126: 107-120.

21. Matoba S, Kang JG, Patino WD, Wragg A, Boehm M, et al. (2006) p53 regulates mitochondrial respiration. Science 312: 1650-1653.

22. Kim JW, Tchernyshyov I, Semenza GL, Dang CV (2006) HIF-1-mediated expression of pyruvate dehydrogenase kinase: a metabolic switch required for cellular adaptation to hypoxia. Cell Metab 3: 177-185. 